\title{
How to Deal with Pediatric Functional Gastrointestinal Disorders
}

\author{
Desale Yacob · Carlo Di Lorenzo
}

Published online: 5 May 2013

(C) Springer Science + Business Media New York 2013

\begin{abstract}
Pain predominant functional gastrointestinal disorders (FGID) are common in the pediatric population and this review article is meant to explore the diagnostic and therapeutic approaches taking into account the most recent developments in the field.
\end{abstract}

Keywords Children - Functional gastrointestinal disorder - Irritable bowel syndrome - Abdominal pain . Anxiety $\cdot$ Depression

\section{Introduction}

Functional gastrointestinal disorders (FGID) as a group are very common in the pediatric population affecting all age groups. Community and school based studies have reported that as many as $13-38 \%$ of children and adolescents experience weekly abdominal pain and up to $24 \%$ of children report symptoms lasting longer than 2 months [1, 2]. Such disorders have a substantial impact on quality of life, socialization, health expenditure and school attendance and may be associated with long-term psychological disturbances [3, 4]. The pathophysiology of FGID is thought to involve abnormalities in the relationship between the enteric and central nervous systems [5]. There is a high prevalence of psychiatric disorders in FGID patients, most notably an increased prevalence of anxiety and depression [6]. The reverse is also true and patients with internalizing psychiatric disorders are found to harbor a higher prevalence of pain predominant FGID [7•].

D. Yacob $(\bowtie) \cdot$ C. Di Lorenzo

Division of Pediatric Gastroenterology, Nationwide Children's Hospital, 700 Columbus Drive, Columbus, OH 43205, USA

e-mail: des.yacob@nationwidechildrens.org
Childhood pain-predominant FGID include several disorders, namely irritable bowel syndrome (IBS), functional dyspepsia (FD), abdominal migraine and functional abdominal pain (FAP). These conditions are all characterized by recurrent episodes of abdominal pain or discomfort in the absence of inflammatory, anatomic, metabolic, or neoplastic processes that explain the symptoms [8]. Although there are some differences related to the underlying pathophysiologic disturbance, they share enough commonalities that they can be discussed in this article as a group.

Primary care providers and gastroenterologists should accept the reality that many of their patients will present with a FGID and need help. A pragmatic and effective diagnostic and therapeutic approach would serve their patients best and have a positive impact on their practices. Symptoms based criteria, such as the "Rome criteria" are becoming the gold standard for diagnosing FGID. The four tenants of therapy include reaching the diagnosis, explaining the disorder, mapping the natural course of the disease, and providing a therapeutic plan based on the biopsychosocial model [9].

\section{Diagnosis}

One of the major hurdles in caring for children with FGID has been the inability to make the diagnosis in a timely manner and explain it convincingly to patients and their parents. This stems from the physician's lack of understanding or appreciation of the illness, further complicated by the absence of reliable biologic markers and specific diagnostic tests. While the advancement of medical technology has greatly impacted how most symptoms are investigated and diseases are diagnosed, extensive testing 
has very little role in the evaluation of patients with FGID. A thorough history and comprehensive physical exam with a limited workup continues to be the primary and most effective approach in evaluating symptoms of FGID.

The physician-patient encounter can be divided into three major stages, with each one playing an essential role in reaching the correct diagnosis and ensuring the visit is fruitful. The three stages, as represented by the three "Ps", are the preparatory, patient encounter and post encounter stages. Each has its own role and importance but they all exist in a continuum and are complementary to each other.

\section{Preparatory Stage}

In preparation to meet the patient the physician should review the vital signs, height and weight plotted on the growth chart, past workup, and a completed pre-visit form. The pre-visit form usually consists of demographics, review of systems, past medical history, surgical history, family and social history. This form, typically completed by a parent before the visit, should be available to the physician prior to entering the exam room. It often provides very helpful information and a few minutes should be spent reviewing it. This enables the physician to move on to the next stage armed with adequate data.

\section{Patient Encounter Stage}

This stage is at the core of the visit and comprises many parts. It begins with the greeting and introduction and concludes with a discussion of a therapeutic plan. History of present illness, physical exam, diagnosis and discussion of any diagnostic workup are also part of this stage. When a physician for whatever reason skips the greeting and introduction part, he may be undermining his ability to provide an effective care by not setting a positive tone at the beginning of the visit which is key to establishing an effective patient-doctor relationship.

The chief complaint of abdominal pain should be taken seriously and fully explored. The onset, location, nature, frequency, duration, alleviating and triggering factors, associated symptoms such as bowel movement changes, nausea, vomiting, early satiety, fever, weight loss, night time awakening, joint involvement and fatigue help in distinguishing organic from functional etiologies. The physical exam follows the history taking but in reality it starts the moment the physician steps into the room. The patient with a FGID is most likely to be well appearing and nutritionally healthy. The abdominal exam will typically demonstrate periumbilical and nonspecific pain without rebound tenderness.

At this point the provider ought to have a good sense of the cause of the abdominal pain and be ready to establish the diagnosis of a FGID. Practitioners have to move away from thinking of FGID as diagnosis of exclusion. This does not, however, mean that the diagnosis is without a differential. On the contrary, the physician should always think of other etiologies that may cause abdominal pain and be willing to consider further tests should the symptoms change in nature or new ones develop. The evidence suggests that it is very unlikely that any diagnostic test will be abnormal when a diagnosis of a FGID is highly suspected. In a retrospective review of 243 patients with pain-predominant FGID, Dhroove et al. [10••] reviewed the workup done as part of the evaluation. None of the diagnostic studies including laboratory tests, upper endoscopy, abdominal X-ray, abdominal ultrasonography, upper gastrointestinal series and abdominal CT scan, resulted in any meaningful abnormality other than nonspecific findings, that were most likely to cause confusion, increase anxiety and lead to more invasive testing and procedures. The argument that normal test results provide a therapeutic benefit is not always true. Bonilla et al. evaluated the impact of normal endoscopies in the clinical outcome of children with pain predominant FGID. They compared a group of children with a FGID who had undergone endoscopic evaluation to a control group with the same diagnosis who were not evaluated by endoscopy. The results showed no difference in the persistence of pain between the two groups [11].

\section{Post Encounter Stage}

At the end of the clinic visit the patient should leave the office with clear instructions and an appointment for a clinic follow up. There is no one single treatment that is guaranteed to work in every circumstance and the various options cannot all be tried at the same time. The patient should be empowered to call if symptoms are not improving in order to discuss other options including referral to a mental health specialist.

\section{Treatment}

The biopsychosocial model is utilized to provide a comprehensive therapeutic plan in the care of patients with FGIDs and is more effective than other approaches. It is a model that takes into account the physical and psychosocial components of the illness and by virtue of its inclusiveness it has transformed how physicians think of these disorders and manage the patients afflicted by them. Psychological and cognitive behavioral therapy, diet manipulation, hypnotherapy, acupuncture, bulking agents, pro- and prebiotics, pharmacotherapy that consist of antispasmodics, tricyclic antidepressants, anti-diarrheals, stool softeners, 
antibiotics, melatonin and chloride channel agonists constitute some of the many interventions that can be tried.

As already mentioned, treatment of the child with a FGID starts at the time of the initial evaluation. It is of great importance to convey the impression that the patient's complaints are being taken seriously. The biomedical model pervasive in the Western societies gives diseases in which inflammation or anatomic changes can be detected more legitimacy than disorders in which it is the function of the organ that is altered. This biased view against illnesses of functional nature is widespread and trickles down to trainees. In a study of physicians on-call, it was found that providers believed phone calls from patients with functional disorders were less serious and less reasonable. Moreover, the providers believed that such patients were less disabled, and liked them less than patients with an organic diagnosis [12]. Many medical providers have a bias towards FGID and rarely recognize them as "real diseases". As a consequence the message that is given to the patients often implies that these conditions are "in their head" and leads to poor therapeutic relationships between patient and physician.

It is essential to give the parents time to speak and explain their concerns. We find the queries "how can I help you?" and "please tell me what brought you here" to be reasonable starting points. In a study in primary care, patients spoke uninterrupted for an average time of $12 \mathrm{~s}$ after the resident entered the room. One-fourth of the time, residents interrupted patients before they finished speaking [13]. Families have often waited weeks before the clinic appointment and have thought in great detail about what to tell the physician. They deserve to have a few uninterrupted minutes to express their concerns and thoughts before being asked specific questions. Indeed, allowing completion of the opening statement is considered a key component of good physician-patient interaction, as outlined by the Kalamazoo Consensus document [14]. A very recent review by Dr. Drossman [15] summarizes the other key verbal and nonverbal components of optimal communication between a medical provider and the patient. Since a positive physician-patient interaction has been associated with fewer return visits for adults with IBS [16], every physician involved in the care of patients with functional disorders should be familiar with and endorse such behaviors.

In pediatrics, the initial clinic visit is complicated by the need to interact with both the parents and the patient. When parents bring their children to a physician for evaluation of a pain-predominant disorder they often have a set of preconceived notions of the possible causes of their child's problem. Their expectation is that medical providers will expeditiously perform diagnostic tests, determine the "real" cause of the pain, and direct an intervention to quickly and permanently resolve the problem. Fears of serious underlying diseases, such as cancer, often lead parents to ask for extensive testing. Rarely do parents believe that psychosocial issues may be relevant in contributing to their child's symptom and, even when they do, they are usually reticent about discussing them for fear that the physician will "stop looking for the real cause" of the symptoms. On the other hand, the children frequently relate that they do not know why they are being evaluated and are fearful of invasive or painful testing. We find that asking "what do you think is going on?" or "what are you most concerned about?" to be very helpful in directing the amount and type of testing needed. Tailoring the choice of tests to undo parental fears can be an effective way to establish a close therapeutic alliance with the family. In some cases, such as when the fears are totally irrational (for example, being afraid of a bowel obstruction or being concerned about pancreatic cancer in an otherwise totally healthy child) a quick discussion of why this is exceedingly unlikely may be reassuring enough that no further test is needed. Table 1 summarizes practical points that may improve the quality of the first encounter with the family of a patient with a FGID and positively impact the outcome. Due to the high rate of symptomatic success of reassurance, medications are not necessary for most patients with a FGID and should be reserved for patients with symptoms interfering with age appropriate activities and impacting quality of life. The main goal of the therapy for children with a FGID is to reestablish a normal daily life for both the patient and the family and the rest of this review outlines strategies to achieve this goal.

\section{Reassurance and Placebo}

Effective reassurance is very successful in every functional disorder. In order for a reassurance to be truly effective though, parents' and child's fears need to be satisfactorily addressed. No improvement can be expected if the family worries about something else happening every time the child experiences pain. A recent study investigated whether catastrophic thinking about pain by children with functional abdominal pain or by their parents is associated with worse health outcomes [17]. The authors found that child catastrophizing predicted child depression, anxiety and functional disability. Parents catastrophizing led to responses to their children's abdominal pain in ways that encouraged illness behavior. These findings suggest that the perception of threat associated with the symptom may have therapeutic implications in the clinical setting. An explanation about the benign nature of the pain, without trivializing it, is often all that it takes to reduce disability.

The family should be discouraged from reinforcing the symptoms by allowing the child to miss school. Patients 
Table 1 DOs and DON'Ts of visits with patients with FGID

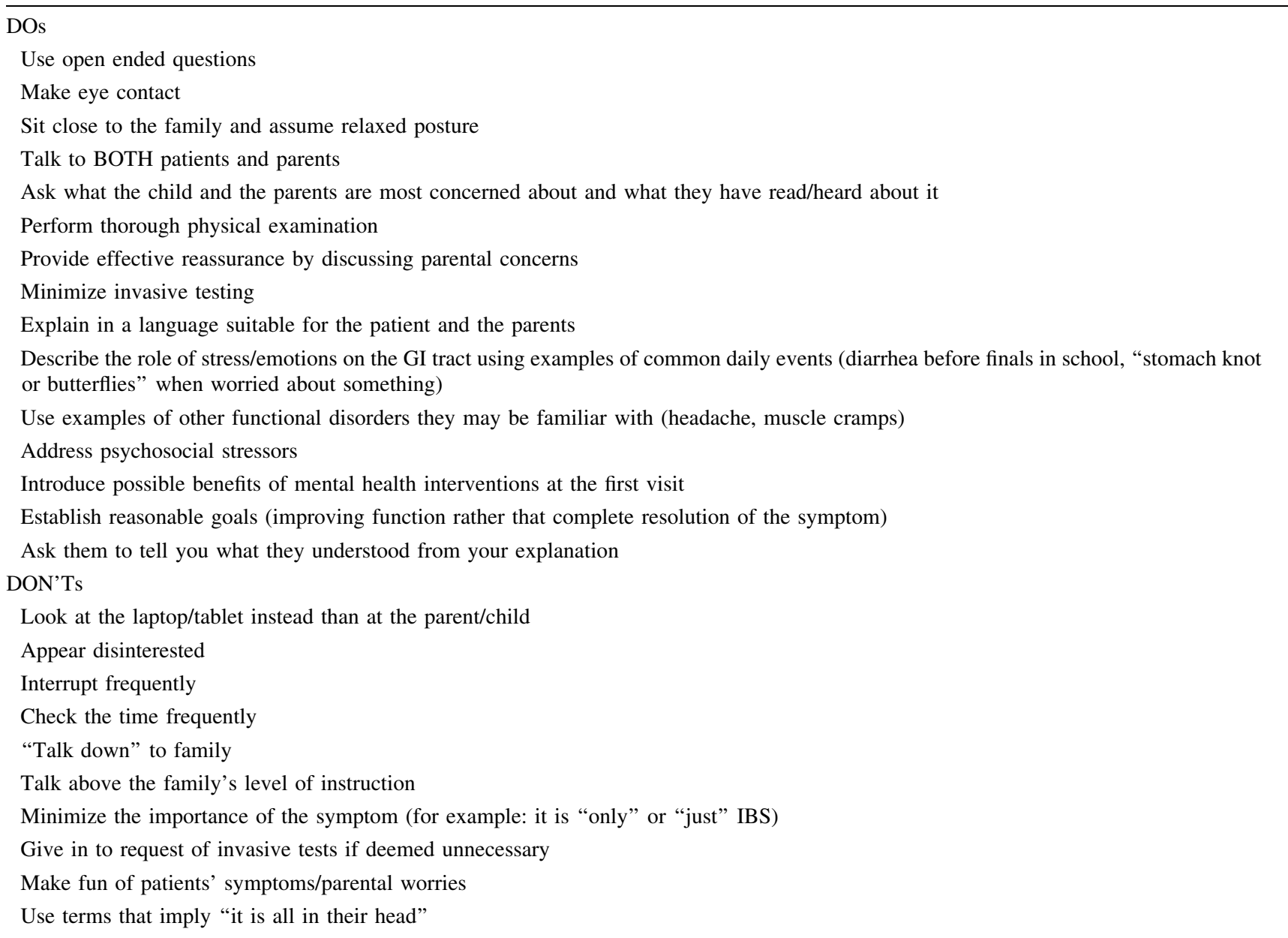

who struggle in academics and have low self-esteem may find the avoidance of school obligation as an unintended benefit of the pain experience [18]; meanwhile, patients who are high achievers may find falling behind in their school assignments discouraging. Children in school are more distracted and less focused on their symptoms, and going to school even while experiencing some pain, rather than being interpreted as unfair or cruel, should instead be interpreted as being integral part of the treatment plan.

The medical provider should point out that FGID are very common and have an overall favorable prognosis. Using terms like IBS, which parents may have heard before, is more helpful than relying on terms such as dyspepsia or hyperalgesia which may be more challenging to interpret. Comparing FGID to headaches, another common disorder that most people are familiar with, may help put symptoms in perspective. The role of anxiety, stress and the importance of the brain-gut axis in the pathophysiology of symptoms should be explained. Instead of falling victim to the dichotomy of body versus brain, medical providers should shift the focus of their explanation on the widely recognized effects that emotions have on gastrointestinal functions, and emphasize that such effects are even more pronounced in patients suffering from FGID.

Finally, the provider should make clear that the child should be an active participant in his or her recovery. A passive coping style has been found to result in long-term disability in children with FAP [19]. The patient needs to be "ready to improve". Assuming a sick role is often reinforced by the use of social media. We have encountered many children who, after being hospitalized for evaluation of their mysterious ailment, have become local celebrities due to their active use of blogs, Twitter and Facebook. Such patients, usually adolescents, receive a great deal of sympathy from their friends and may not be "ready to let go", once the functional nature of their symptom is explained to them.

Improvement of symptoms has been reported in up to $60 \%$ of children with functional abdominal pain treated with placebo. It is obviously unethical to administer a placebo to children without informing them that no active drug is given. On the other hand, a recent study in adults 
with IBS randomized patients to either open-label placebo pills presented as "placebo pills made of an inert substance, like sugar pills, that have been shown in clinical studies to produce significant improvement in IBS symptoms through mind-body self-healing processes" or notreatment controls. The authors concluded that even placebos administered without deception constituted an effective treatment for IBS [20].

\section{Addressing Mental Health Disorders}

In a study done in a primary care setting, children with FAP were found to be significantly more likely to receive a diagnosis of an anxiety or a depressive disorder, and displayed higher levels of temperamental harm avoidance, and functional impairment than control subjects. Anxiety disorders were significantly more likely to precede the development of chronic abdominal pain [6]. In another very recent study conducted in the Netherlands, it was found that in one-third of children presenting to general practice for evaluation of abdominal pain, anxiety and depressive problems persisted during 1 year of follow-up [21]. Thus, it behooves the pediatrician to appropriately address the mental health disorder of the child presenting with a FGID if a true positive outcome is to be achieved. We find that introducing the role of a psychologist or a psychiatrist as an integral component of the treatment rendered to children with chronic pain at the time of the initial encounter is helpful to prepare the parents and the child to the fact that such a specialist may in the future be involved in the care of the child. It also minimizes the risk that referral to a mental health specialist will be seen as abandonment by the physician who has followed the child up to that point.

\section{Diet}

There is no specific diet that is universally effective for children with FGIDs. A detailed dietary history may identify factors that patients may feel as aggravating or provoking their symptoms. A Cochrane review [22] identified two trials, including 83 participants, comparing fiber supplements with placebo and two trials, including 90 participants, comparing lactose-containing with lactosefree diets. It was concluded that there was no evidence that fiber supplements or lactose-free diets were effective in the management of children with intermittent or chronic abdominal pain. Emerging evidence indicates that the consumption of fermentable oligosaccharides, disaccharides, monosaccharides and polyols (FODMAP) may aggravate symptoms in some patients with IBS. While there are no data yet on the efficacy of such intervention in children, there have been well designed studies in adults with IBS which have suggested that a low FODMAP diet is more effective than standard dietary advice for symptom control in IBS [23••]. It has been hypothesized that FODMAP affect hydrogen production in the intestine, influence the amount of methane produced, and modulate gastrointestinal and systemic symptoms experienced by patients with IBS [24].

\section{Tricyclic Antidepressants}

Tricyclic antidepressants (TCA) have been used successfully in the treatment of multiple chronic pain syndromes [25-28]. In addition to their action on noradrenergic and serotoninergic receptors, TCA have antimuscarinic and antihistaminic effects and can stimulate neurogenesis, neural remodeling, and synaptic plasticity in the hippocampus [29]. The analgesic effects of TCA are independent of their effects on depression, and this information should be shared with the family and the patient. When aiming at improving symptoms in FGID, TCA are used at smaller doses than needed for treatment of clinical depression and may require several weeks to provide their beneficial effect. These agents are best administered at bedtime and are especially helpful in patients with diarrhea-predominant IBS and in those with sleep difficulties in whom slowing intestinal transit and the side effects of sleepiness may be of therapeutic value. Three pediatric studies have evaluated the benefit of TCA in children with FGID. A small randomized, placebo controlled trial found that amitriptyline improved quality of life and pain in adolescents with IBS [30]. A larger multicenter, randomized, double-blind trial evaluated the efficacy of amitriptyline on 90 children with FAP or IBS [31]. It showed improvement in $59 \%$ of the children receiving amitriptyline with a similar benefit noted also in the control group receiving placebo. Both groups of children had comparable improvement in pain, disability, depression and somatization scores during the 4 weeks of the trial. Another openlabel study followed 98 pediatric patients who took a TCA for a FGID and found that $78 \%$ responded to the treatment for an average of 10.7 months [32]. It has been suggested that EKG monitoring should be performed before starting the treatment with TCA in children with FGID [33].

\section{Alternative and Complementary Therapy}

A very effective therapeutic strategy for children with FGID involves the use of hypnotherapy or psychotherapy. Hypnotherapy has been shown to be effective in the treatment of both gastrointestinal symptoms and extra-intestinal symptoms that are often associated with IBS in adults [34]. A recent systematic review examined three randomized controlled trials comparing gut-directed hypnotherapy to a control treatment in children with FAP or IBS, with sample 
sizes ranging from 22 to 52 children [35]. All studies showed statistically significant improvement in abdominal pain scores among children receiving hypnotherapy. It was concluded that the therapeutic effect of hypnotherapy is superior to standard medical care in children with FAP or IBS. In order to obviate the common problem of lack of access to mental health providers trained to deliver hypnotherapy, one of the studies tested a home-based, guided imagery treatment protocol. The authors used audio and video recordings, which were inexpensive, easy for health care professionals and patients to use, and applicable to a wide range of health care settings [36•]. Benefit of the treatment lasted for at least 6 months. Another study evaluated the use of Internet based cognitive behavioral therapy in children with a variety of chronic pain syndromes, including abdominal pain [37]. The treatment group completed 8 weeks of online modules including relaxation training, cognitive strategies, parent operant techniques, communication strategies, and sleep and activity interventions. The study concluded that Internet delivery of family cognitive behavioral treatment is efficacious and widely acceptable for reducing pain and improving function among children and adolescents with chronic pain.

\section{Probiotics}

Despite evidence that changes in enteric flora may play a role in childhood IBS [38••], convincing evidence for a pathogenic role of bacterial overgrowth or for a beneficial effect of probiotics or antibiotic therapy in pediatrics is still limited. Three well designed pediatric studies using different study protocols evaluated the role of different probiotics in the treatment of children with pain-predominant FGID and were reviewed in a recent meta-analysis [39]. It was concluded that the use of Lactobacillus rhamnosus GG moderately increases treatment success in children with pain-predominant FGID, particularly among children with IBS. In an international, multicenter, randomized, doubleblind, placebo-controlled, crossover study, children 4-18 years of age with IBS were randomized to receive either VSL\#3 (a probiotic preparation containing 8 strains of bacteria) or placebo for 6 weeks [40]. VSL\#3 was found to be more effective than placebo in ameliorating symptoms and improving the quality of life. Despite these promising results, there is currently little agreement on when, for how long, and which probiotic to use in children with FGID.

\section{Treating the Parents}

Parental responses to the child complaint of pain may lead to either persistence or resolution of the symptom. Parents can maximize wellness behaviors in their children by displaying appropriate responses and rewarding healthy adaptive behaviors. In a brilliant clinical study by Walker et al. [41] parents were trained to interact with their children who were experiencing abdominal discomfort in one of three ways: attention, distraction, or no instruction. Symptom complaints by either children with chronic abdominal pain or well children nearly doubled in the attention subgroup and were reduced by half in the distraction subgroup. Dr. Levy and coworkers [42••] tested the efficacy of an intervention designed to improve outcomes in childhood abdominal pain by altering parental responses to pain and children's ways of coping and thinking about their symptoms. They found that children in the treatment group showed greater decreases in pain and symptom severity. Parents in the cognitive-behavioral condition displayed greater decreases in solicitous responses to their child's symptoms.

\section{Others}

In a recent and somewhat surprising study of 200 children with recurrent abdominal pain referred to secondary care, laxative therapy was successful in $46 \%$, resulting in nearly all patients with functional abdominal pain to become pain free [43].

Cyproheptadine is a medication widely used in children with dyspeptic symptoms, poor appetite and as prophylaxis of cyclic vomiting and abdominal migraine episodes. A double-blind, placebo-controlled trial of cyproheptadine in the treatment of 29 children with FAP found that $86 \%$ of children in the cyproheptadine group and $36 \%$ of those in the placebo group had improvement or resolution in selfreported frequency and duration of abdominal pain [44]. In a very recent retrospective study of 80 children, cyproheptadine was found to be safe and effective for treating dyspeptic symptoms in children, particularly in young children and those with vomiting soon after eating and with retching after fundoplication [45].

Montelukast is a leukotriene receptor antagonist commonly used in the maintenance treatment of asthma. Based on the finding of duodenal eosinophilia in children with FD, an initial double-blind, randomized, placebo-controlled, cross-over study and a subsequent open-label study evaluated the benefit of montelukast in school-age children presenting with postprandial symptoms consistent with FD. In both studies there seemed to be a significant clinical benefit in children receiving montelukast [46, 47].

\section{Natural History}

Children with abdominal pain have been found to have an increased risk of psychiatric disorders in adulthood [48, 
49]. Another study which prospectively followed youth with FAP for up to 15 years found that they may be at increased risk for chronic pain and headache [50]. The most long lasting benefit among the different treatments for childhood FGID seems to be the gut-directed hypnotherapy. It has been reported that two-thirds of children with FAP or IBS are still in remission almost 5 years after treatment, making it a highly valuable therapeutic option [51]. A brief intervention to reduce parental solicitousness and increase coping skills suggests a 12-month benefit [52]. It has been reported that the presence of non-GI somatic symptoms in children with FAP identifies a group of patients at higher risk to meet criteria for FGID later in life [53]. Obesity has been associated with poor outcome and disability at long-term follow-up in children with abdominal pain-related FGIDs [54].

\section{Conclusions}

In conclusion, there are many effective ways to deal with the child presenting with FGID. The initial clinic visit as divided in three complementary stages facilitates effective diagnostic and therapeutic intervention. It is often the comfort level of the medical provider in addressing functional disorders that has the biggest impact on outcome. Physicians who feel discouraged and powerless in dealing with a child in whom no organic disease can be found will be less likely to be helpful than providers who have a good understanding of the multifaceted aspects of FGID and who embrace the biopsychosocial model of health care delivery.

Disclosure Desale Yacob and Carlo Di Lorenzo declare no conflict of interest.

\section{References}

Papers of particular interest, published recently, have been highlighted as:

- Of importance

- Of major importance

1. Hyams JS, Burke G, Davis PM, et al. Abdominal pain and irritable bowel syndrome in adolescents: a community-based study. J Pediatr. 1996;129:220-6.

2. Saps M, Seshadri R, Sztainberg M, et al. A prospective schoolbased study of abdominal pain and other common somatic complaints in children. J Pediatr. 2009;154:322-6.

3. Neal KR, Hebden J, Spiller R. Prevalence of gastrointestinal symptoms six months after bacterial gastroenteritis and risk factors for development of the irritable bowel syndrome: postal survey of patients. BMJ. 1997;314:779-82.

4. Hulisz D. The burden of illness of irritable bowel syndrome: current challenges and hope for the future. J Manag Care Pharm. 2004;10:299-309.
5. Ringel Y, Sperber AD, Drossman DA. Irritable bowel syndrome. Annu Rev Med. 2001;52:319-38.

6. Campo JV, Bridge J, Ehmann M, et al. Recurrent abdominal pain, anxiety, and depression in primary care. Pediatrics. 2004;113: 817-24.

7. - Yacob D, Di Lorenzo C, Bridge JA, et al. Prevalence of painpredominant functional gastrointestinal disorders and somatic symptoms in patients with anxiety or depressive disorders. J Pediatr. 2013. doi:10.1016/j.jpeds.2013.02.033. This study highlights the importance of the association between internalizing psychiatric disorders and FGID.

8. Rasquin A, Di Lorenzo C, Forbes D, et al. Childhood functional gastrointestinal disorders: child/adolescent. Gastroenterology. 2006;130:1527-37.

9. Hyman PE, Monagas J. Rectal perceptual hypersensitivity: a biomarker for pediatric irritable bowel syndrome. J Pediatr. 2010; 156:5-7.

10. $\bullet$ Dhroove G, Chogle A, Saps M. A million-dollar work-up for abdominal pain: is it worth it? J Pediatr Gastroenterol Nutr 2010, 51:579-583. This paper shows that extensive testing in patients who receive a diagnosis of FGID based on history and physical examination is not warranted.

11. Bonilla S, Wang D, Saps M. The prognostic value of obtaining a negative endoscopy in children with functional gastrointestinal disorders. Clin Pediatr (Phila). 2011;50:396-401.

12. Dalton CB, Drossman DA, Hathaway JM, Bangdiwala SI. Perceptions of physicians and patients with organic and functional gastrointestinal diagnoses. Clin Gastroenterol Hepatol. 2004;2: 121-6.

13. Rhoades DR, McFarland KF, Finch WH, Johnson AO. Speaking and interruptions during primary care office visits. Fam Med. 2001;33:528-32.

14. Makoul G. Essential elements of communication in medical encounters: the Kalamazoo consensus statement. Acad Med. 2001;76:390-3.

15. Drossman DA. 2012 David Sun lecture: helping your patient by helping yourself-how to improve the patient-physician relationship by optimizing communication skills. Am J Gastroenterol. 2013;108:521-8.

16. Owens DM, Nelson DK, Talley NJ. The irritable bowel syndrome: long-term prognosis and the physician-patient interaction. Ann Intern Med. 1995;122:107-12.

17. Langer SL, Romano JM, Levy RL, et al. Catastrophizing and parental response to child symptom complaints. Child Health Care. 2009;38:169-84.

18. Walker LS, Claar RL, Garber J. Social consequences of children's pain: when do they encourage symptom maintenance? J Pediatr Psychol. 2002;27:689-98.

19. Walker LS, Smith CA, Garber J, Claar RL. Testing a model of pain appraisal and coping in children with chronic abdominal pain. Health Psychol. 2005;24:364-74.

20. Kaptchuk TJ, Friedlander E, Kelley JM, et al. Placebos without deception: a randomized controlled trial in irritable bowel syndrome. PLoS One. 2010;5:e15591.

21. Gieteling MJ, Lisman-van Leeuwen Y, Passchier J, et al. The course of mental health problems in children presenting with abdominal pain in general practice. Scand J Prim Health Care. 2012;30:114-20.

22. Huertas-Ceballos AA, Logan S, Bennett C, Macarthur C. Dietary interventions for recurrent abdominal pain and irritable bowel syndrome in childhood. Cochrane Database Syst Rev. 2009;21: CD003019.

23. • Staudacher HM, Whelan K, Irving PM, Lomer MC. Comparison of symptom response following advice for a diet low in fermentable carbohydrates (FODMAPs) versus standard dietary advice in patients with irritable bowel syndrome. J Hum Nutr 
Diet 2011,24:487-495. This paper studies the benefit of a dietary therapy with low fermentable products in IBS and shows that this option represents a promising therapeutic option.

24. Ong DK, Mitchell SB, Barrett JS, et al. Manipulation of dietary short chain carbohydrates alters the pattern of gas production and genesis of symptoms in irritable bowel syndrome. J Gastroenterol Hepatol. 2010;25:1366-73.

25. Dekel R, Drossman DA, Sperber AD. The use of psychotropic drugs in irritable bowel syndrome. Expert Opin Investig Drugs. 2013;22:329-39.

26. Perrot S, Javier RM, Marty M, et al. Antidepressant use in painful rheumatic conditions. Rheum Dis Clin North Am. 2008;34:433-53.

27. Romano TJ. Fibromyalgia in children; diagnosis and treatment. W V Med J. 1991;87:112-4.

28. Fishbain D. Evidence-based data on pain relief with antidepressants. Ann Med. 2000;32:305-16.

29. Han X, Tong J, Zhang J, Farahvar A, Wang E. Imipramine treatment improves cognitive outcome associated with enhanced hippocampal neurogenesis after traumatic brain injury in mice. J Neurotrauma. 2011;28:995-1007.

30. Bahar RJ, Collins BS, Steinmetz B, Ament ME. Double-blind placebo-controlled trial of amitriptyline for the treatment of irritable bowel syndrome in adolescents. J Pediatr. 2008;152:685-9.

31. Saps M, Youssef N, Miranda A, et al. Multicenter, randomized, placebo-controlled trial of amitriptyline in children with functional gastrointestinal disorders. Gastroenterology. 2009;137:1261-9.

32. Teitelbaum JE, Arora R. Long-term efficacy of low-dose tricyclic antidepressants for children with functional gastrointestinal disorders. J Pediatr Gastroenterol Nutr. 2011;53:260-4.

33. Patra KP, Sankararaman S, Jackson R, Hussain SZ. Significance of screening electrocardiogram before the initiation of amitriptyline therapy in children with functional abdominal pain. Clin Pediatr (Phila). 2012;51:848-51.

34. Houghton LA, Whorwell PJ. Symptomatology, quality of life and economic features of irritable bowel syndrome-the effect of hypnotherapy. Aliment Pharmacol Ther. 1996;10:91-5.

35. Rutten JM, Reitsma JB, Vlieger AM, Benninga MA. Gut-directed hypnotherapy for functional abdominal pain or irritable bowel syndrome in children: a systematic review. Arch Dis Child. 2013;98:252-7.

36. - van Tilburg MA, Chitkara DK, Palsson OS, et al. Audiorecorded guided imagery treatment reduces functional abdominal pain in children: a pilot study. Pediatrics 2009, 124:e890-e897. A very important study which demonstrate the benefit of a simple, child-and family-friendly cognitive behavioral intervention in the treatment of children with functional abdominal pain.

37. Palermo TM, Wilson AC, Peters M, et al. Randomized controlled trial of an Internet-delivered family cognitive-behavioral therapy intervention for children and adolescents with chronic pain. Pain. 2009;146:205-13.

38. • Saulnier DM, Riehle K, Mistretta TA, et al. Gastrointestinal microbiome signatures of pediatric patients with irritable bowel syndrome. Gastroenterology 2011, 141:1782-1791. This is the first study which highlights differences in microbiome between children with IBS and control individuals. It has important diagnostic and therapeutic implications, suggesting that modification of colonic flora with probiotics or antibiotics may be of benefit to a subgroup of children with FGID.
39. Horvath A, Dziechciarz P, Szajewska H. Meta-analysis: Lactobacillus rhamnosus GG for abdominal pain-related functional gastrointestinal disorders in childhood. Aliment Pharmacol Ther. 2011;33:1302-10.

40. Guandalini S, Magazzù G, Chiaro A, et al. VSL\#3 improves symptoms in children with irritable bowel syndrome: a multicenter, randomized, placebo-controlled, double-blind, crossover study. J Pediatr Gastroenterol Nutr. 2010;51:24-30.

41. Walker LS, Williams SE, Smith CA, et al. Parent attention versus distraction: impact on symptom complaints by children with and without chronic functional abdominal pain. Pain. 2006;122:43-52.

42. •• Levy RL, Langer SL, Walker LS, et al. Cognitive-behavioral therapy for children with functional abdominal pain and their parents decreases pain and other symptoms. Am J Gastroenterol 2010, 105:946-956. This study is of high importance given the nature of pediatric FGID and the importance of parental involvement. Children's ability to cope with pain and maintain age-appropriate function is modified by the parental input.

43. Gijsbers CF, Kneepkens CM, Schweizer JJ, et al. Recurrent abdominal pain in 200 children: somatic causes and diagnostic criteria. Acta Paediatr. 2011;100:e208-14.

44. Sadeghian M, Farahmand F, Fallahi GH, Abbasi A. Cyproheptadine for the treatment of functional abdominal pain in childhood: a double-blinded randomized placebo-controlled trial. Minerva Pediatr. 2008;60:1367-74.

45. Rodriguez L, Diaz J, Nurko S. Safety and efficacy of cyproheptadine for treating dyspeptic symptoms in children. J Pediatr. 2013. doi:10.1016/j.jpeds.2012.12.096.

46. Friesen CA, Kearns GL, Andre L, et al. Clinical efficacy and pharmacokinetics of montelukast in dyspeptic children with duodenal eosinophilia. J Pediatr Gastroenterol Nutr. 2004;38:343-51.

47. Friesen CA, Neilan NA, Schurman JV, et al. Montelukast in the treatment of duodenal eosinophilia in children with dyspepsia: effect on eosinophil density and activation in relation to pharmacokinetics. BMC Gastroenterol. 2009;11(9):32.

48. Campo JV, Di Lorenzo C, Chiappetta L, et al. Adult outcomes of pediatric recurrent abdominal pain: do they just grow out of it? Pediatrics. 2001;108:E1.

49. Hotopf M, Carr S, Mayou R, et al. Why do children have chronic abdominal pain, and what happens to them when they grow up? Population based cohort study. BMJ. 1998;316:1196-200.

50. Walker LS, Dengler-Crish CM, Rippel S, Bruehl S. Functional abdominal pain in childhood and adolescence increases risk for chronic pain in adulthood. Pain. 2010;150:568-72.

51. Vlieger AM, Rutten JM, Govers AM, et al. Long-term follow-up of gut-directed hypnotherapy vs. standard care in children with functional abdominal pain or irritable bowel syndrome. Am J Gastroenterol. 2012;107:627-31.

52. Levy RL, Langer SL, Walker LS, et al. Twelve-month follow-up of cognitive behavioral therapy for children with functional abdominal pain. JAMA Pediatr. 2013;167:178-84.

53. Dengler-Crish CM, Horst SN, Walker LS. Somatic complaints in childhood functional abdominal pain are associated with functional gastrointestinal disorders in adolescence and adulthood. J Pediatr Gastroenterol Nutr. 2011;52:162-5.

54. Bonilla S, Wang D, Saps M. Obesity predicts persistence of pain in children with functional gastrointestinal disorders. Int J Obes (Lond). 2011;35:517-21. 